\title{
Research on Japanese Teaching in the Perspective of Contrastive Linguistic
}

\author{
Rui Zhang \\ College of Oriental Languages, Harbin Normal University, Harbin, \\ 150015, China \\ Capital University of Tokyo, Tokyo, 1920364, Korea
}

\begin{abstract}
With the deepening of economic globalization and the ties between countries around the world are closer, many Chinese universities have set up a Japanese professional to cultivate the Japanese top students. In the process of teaching Japanese, the first thing to do is to innovate and reform the traditional Japanese teaching methods, so that the best teaching results to achieve smooth in a certain period of time. This paper discusses the topic of Japanese teaching from the perspectives of comparative linguistics, and analyzes Japanese teaching from the perspective of comparative linguistics such as Japanese vocabulary teaching and vocabulary contrast, Japanese phonetics teaching and phonetic contrast, Japanese grammar teaching and grammar comparison, and Japanese translation teaching and pragmatic contrast.

Keywords comparative linguistics, Japanese, teaching:
\end{abstract}

\section{Introduction}

As a branch of modern linguistics, comparative linguistics is to compare the similarities and differences between the two languages and the purpose is to solve the translation or teaching problems. The contrastive study includes vocabulary, phonetics, pragmatics, semantics, grammar, and so on. The comparative study can be carried out from the perspective of national, psychological and cultural aspects of language, and can also be compared with punctuation systems in 
different languages. In the process of globalization, more and more scholars attach importance to linguistics.

\section{Japanese vocabulary teaching and vocabulary contrast}

In the language, the smallest can be used independently of the combination of sound and meaning is the word, as a collection of words in the language, the study of words from the meaning and form of the word two aspects. The word has a very complex meaning, which can reflect the human emotions, but also to reflect human cognition, both the possibility of connecting the language of the external world, but also linked to the possibility of internal function of the language. The word can be divided into the emotional meaning of the language and the language, in the cognitive aspect can be divided into the intra and extragrammatical meaning.

The similarities between Chinese and Japanese vocabularies are achieved by the common history and culture of the two countries, but there are many differences between them in the process of the passage of time and the advance of history.

In the expression of the same meaning, the Japanese language is usually applied to different things, its performance in the same things, often have different performance implications. The reason for this is that China and Japan have different semantic scope and cognition.

In contrast to the Japanese and Japanese vocabulary, we can see that at the beginning of the study of Japanese, we should not give too much explanation. During the middle of vocabulary teaching, we will explain the appropriate Japanese culture, Japanese translation, Japanese extensive reading and intensive reading. There are some overlapping words in China and Japan, while others are different. For example, the word "opinions" has the same meaning in both China and Japan, that is, the views and ideas on things. In Chinese, "opinion" contains the meaning of criticism, blame and dissatisfaction, but in Japanese does not have this meaning, in Japanese opinion also contains a verb usage, the meaning of advice, advice and so on. From this we can see that one of Chinese and Japanese has a broader meaning of the word. For example, the word "character" in Japanese means the nature and characteristics of things and people. In Chinese, they are only used to describe people. "Tension" in the Japanese word contains two meanings, the relationship between instability and physical and mental tension, in addition to the above two meanings, but also contains the lack of material and difficult and intense, busy these two meanings. In the comparison of Chinese and Japanese vocabulary research results and the appropriate introduction, can make the students of Japanese and its cultural connotations, etc. easier to understand. 


\section{Japanese phonetics teaching and phonetic contrast}

As the basic method of language expression and material surface, the study of speech needs to be based on the full understanding of the nature of language, first of all need to understand the speech characteristics of the language. Different languages have different regional, national and phonological characteristics. Therefore, in the process of comparing Chinese and Japanese, they can be carried out from the three aspects of intonation, tone and phoneme.

The number of phonemes in Chinese is much larger than that in Japanese. Japanese phonemes are basically included in Chinese phonemes, but the pronunciation methods of Japanese and Chinese are different.

Chinese and Japanese are all high and low tone, but with different levels of change in position, the Japanese are generally different meanings through the different syllable changes in the performance of the Chinese are generally different meanings through different syllable changes in performance.

There are different levels of tones in the sentence and this level of change is the tone, which has a semantic sense of proportion, emotional expression and tone to distinguish the role. In Japanese and Chinese, the change of intonation is usually expressed by different emotional, meaning, and mood changes at the end of the sentence. However, in the Japanese language, each of its internal sound does not have a high and low changes, a phrase or word only to allow the existence of a pitch change, and its changes must be from high to low, the tone will change with the word shape And tends to flat. The analysis of the whole sentence of the Japanese sentence reveals that it has a relatively flat pitch, and it is not all syllables are high and low changes, the change is not so obvious in Chinese, so compared to Chinese, Japanese sounds more peaceful, And the role of intonation in Chinese is far greater than the Japanese.

Comparing Chinese and Japanese phonetics can help students to understand how to distinguish similar pronunciations in Japanese and Chinese, as well as Chinese pronunciation that is not available in Japanese. This contrast can be applied at the beginning of learning Japanese pronunciation. For example, Class. Chinese and Japanese have five vowels, but relative to Chinese, Japanese has a smaller mouth shape changes, which have different tuning methods and parts. For example, the syllable "ya" in Chinese has four tones, including duck (flat sound), teeth (two), elegant (three), rolling (four), but the level of change in Japanese pronunciation is not Exist, does not have a meaning. In Japan, the level of a single scale changes in Japanese does not have a meaning, but its meaning in Chinese there are many. In addition, the Japanese and Chinese have different ranges and the range of Chinese is much wider than the Japanese, so many Japanese people to hear the Chinese people speak, always mistakenly think they are quarreling. In distinguishing and listening to scales, it is necessary for students to have a better grasp of the pronunciation based on the lack of knowledge of phonetic theory in order to distinguish the similar pronunciations, so that the students can better understand the Japanese intonation and pronunciation of the accepted. 


\section{Japanese grammar teaching and grammar contrast}

Grammar has a relatively broad concept in the text discussed in the concept of grammar is a narrow sense of the concept. In Chinese and Japanese, the sentence composition needs to observe certain rules, and the "main + predicate + object" is the basic Chinese word order. In Japanese, the "main + object + predicate" is the basic word order, so the lattice auxiliary word is indispensable in the Japanese sentence, which can form the object and the main grid, the predicate part is the subject and core of the sentence, Constitute Japanese, there are many changes in the use of which exists, but does not exist in the Chinese language.

The reason is that these students have a good understanding of Japanese, and their ability of discrimination and reception is also good, and has a certain depth of grammar level, with a high context of the Japanese students. Requirements, so this is in line with the needs of senior students. For example, in Japanese, "は本 読むむががががとががきです" means "I like reading books". As can be seen from this one sentence, "格" is a very obvious grammatical category in Japanese. After the change is made, a past tense, a completed body, a continuum, and the like can be formed. In the Chinese language, you can use the function words to express the voice and tense. For example, the addition of "a" can be past and complete, and so expressed. In the way of expressing modality, Japanese can be achieved by adding auxiliary verbs, mood auxiliary particles and inflection, such as "でしょう" "ne", etc., the completion of Chinese is also achieved through the use of the word. So you need to compare the Japanese grammar, you can make the students use the Japanese environment and occasions to better understand.

\section{Japanese translation teaching and pragmatic contrast}

In the process of expression, the Sino-Japanese language because of the existence of different environments, so their understanding is different, but also lead to cultural differences. In addition to the above contrasting differences, the differences between Chinese and Japanese in the field of pragmatics are also very important, and the differences are mainly analyzed from three aspects.

Contrast style. Oral and written language is the expressions of the two languages exist. In Japanese, its written expression is "Chinese", and its oral expression is "and language". In Chinese, the division is not so clear, so Chinese in many cases, Japanese expression will be directly grafted Chinese expression. So it is not the context at the time as the goal, the style of change to be ignored, so there will be misuse of the situation.

In language research, language sense is not a technical term and its definition is not clear, but in the surrounding semantics, language sense in the session when its role is very subtle. Due to subtle differences in language sense, the same word contains the meaning will be different. 
Due to the different cultural backgrounds, the same vocabulary of the colour will be different. For these different expressions, if not pay more attention, there will be many unnecessary misunderstandings.

Compared with the Japanese use, can help students to understand the hidden meaning of the Japanese article better, which can help teachers to a large extent, Japanese translation and discourse teaching, it is also more suitable for high school students, high-grade students but also should gradually grasp the hidden meaning of the article. For example, in Chinese, "because", "because" and so can express the meaning of the reason, but its no Chinese style differences in Japanese among the written language and spoken, "ゆえ" and "ta め" as "cause" Of the written language, "の で" for the spoken language. From this point can also be seen due to differences in style, there is not the same meaning. For example, in Japanese, the term "self-love" is often used at the end of the epistolary, which is a derogatory term used in Chinese to denote self-respect, but in Japanese there is no derogatory meaning. The word "profound" does not have an emotional bias in Chinese, and the phrases used in it are "profound analysis", but the term is derogatory in Japanese and generally expresses a serious and serious meaning. "Stubborn" is generally used to describe the character of the person, in Chinese means stubborn and tone heavier, in Japanese means stubborn, lighter tone. Differences in praise and colour, allowing students to better grasp the author's point of view.

The contrast between the strength of language sense, you need the students in the translation process, the "letter, up, Ya," these three points to do. Appropriate introduction of pragmatic contrast can enable students to understand the profound connotation of the article in the process of processing the article information, so as to understand the author's idea.

\section{Conclusion}

This paper discusses the Japanese teaching from the perspective of comparative linguistics. Only when the foreign language teachers have a good understanding of the causes of errors in the process of learning a foreign language, can these errors be effectively corrected and avoided. The process of learning a foreign language is in fact the process of migrating their habits from the mother tongue to the foreign language. When the foreign language and the mother tongue has some same structure, beneficial migration will occur, and under this premise it can enable students to use the new habits of foreign language; when the foreign language and the mother tongue structure is different, the harmful migration will follow. In the specific comparison of the mother tongue and foreign language, students can understand the reasons for the error. There are many similarities and differences between Chinese and Japanese. After analyzing and contrasting the two from the aspects of vocabulary, pronunciation, grammar and pragmatics, we can find out the differences between them and ensure a better application of contrastive linguistic research in Japanese teaching. 


\section{Acknowledgements}

Heilongjiang Province Higher Education Teaching Reform Project "Innovation and practice exploration of Japanese professional practical talents training mode" (No: JG2012010372); Heilongjiang Provincial Education Department Humanities and Social Science Item "The comparative discourse analysis of Chinese and Japanese disastrous news reports" (12532198).

\section{References}

[1] Zhang Ziru, Wang Chengcheng. Comparative Study of Chinese and Japanese Demonstratives Based on Corpus: A Comparative Study of "コ ・ ソ ・ a" Series and "this". Journal of Xi'an International Studies University, 55(12), pp.68-70, 2011

[2] Chen Mengran. Research on English-Japanese Bilingual Teaching from the Perspective of Contrastive Linguistics. Modern Property, 8 (5), pp.87- 91, 2013

[3] Zhang Yongsheng. Japanese Language Teaching from the Perspective of Contrastive Linguistics, Journal of Henan Mechanical and Eectrical Engineering College, 12(10), pp. 18- 20, 2012

[4] Bu Qingli. The Sociolinguistic Perspective of Japanese and Its Implications for Japanese Teaching, Journal of Hubei Radio \& Teleutson Univer Sity, 9(6), pp.58- 61, 2011

[5] Xiong WeiDu. Linguistic Theory of Memetics under the guidance of the Japanese polysemous words teaching research, 8(4), pp.85-87, 2014 